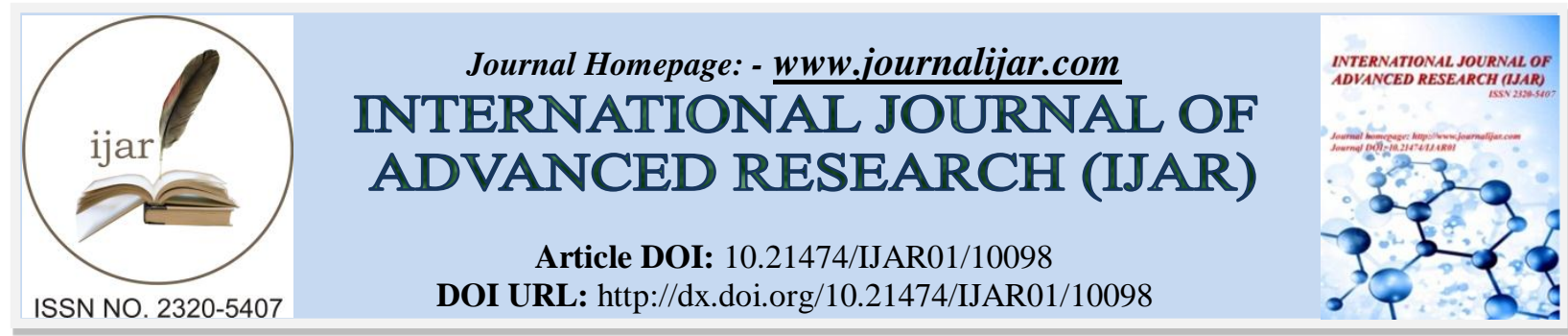

RESEARCH ARTICLE

\title{
ASSESSMENT OF RESULTS OF ARTHROSCOPIC MENISCAL REPAIR IN SOHAG UNIVERSITY HOSPITAL, EGYPT.
}

\section{Khalid A. Abougabal, Moustafa I. Elsayed, Elsayed A. Said, Abdelrahman H. Khalifa and Hassan H. Noaman.}

\section{Manuscript Info}

\section{Manuscript History}

Received: 14 September 2019

Final Accepted: 16 October 2019

Published: November 2019

\section{Abstract}

Background:Knee arthroscopy is the commonest performed orthopedic surgery. Meniscal tears represent a major indication of knee arthroscopy. Comprehensive knowledge and understanding of the structure, function and vascular anatomy of the menisci had opened the door for meniscal repair procedures. Several techniques for meniscal repair do exist. All inside technique is getting more popular use, in this study we report our results of meniscal repair using all inside technique.

Patient and methods:Between January 2015 and June 2019, twenty cases were selected for meniscal repair after thorough clinical, radiological and arthroscopic evaluation. Mean age was 26.5 years (range 20-39). They were 16 males, the main objective was to evaluate the results of arthroscopic meniscal repair using all-inside meniscal repair systems. Clinical evaluation for patients were done according to international knee documentation committee (IKDC).

Results:Mean operative time was $49.75+/-9$ min. Mean IKDC Score before surgery was $40.25+8.35$, while the mean score postoperative was $84+13.92$.

Tear was in the body and posterior third of the meniscus in 15 case, in three cases was on was in the body only while in two cases was in the posterior horn. Second look arthroscopy was performed for cases who showed no improvement in clinical symptoms.

Conclusion:All inside technique provides satisfactory results of meniscal repair, however, we couldn't prove superiority over other techniques of meniscal repair. The essential goal of meniscal repair is protecting the knee from long term degenerative changes by the cushion like and stabilizing effect of the menisci. Having found no difference in surgical outcome between different types of all-inside meniscal repair techniques, we investigated other factors such as age \& sex, injury location, length, zone \& site of tear for their effect.

Patient age and time elapsed from injury was recorded for every case being important factors that might affect repair outcome dramatically. Patient's weight (body mass index) was also recorded as a factor that could jeopardize the repair.

MRI, despite its fallacies, was a major diagnostic tool that helped tear identification as well as exclusion of ligamentous injuries and other associated knee problems. 


\section{Introduction:-}

Knee arthroscopy is the most common type of orthopaedic surgery performed in the United States. Of these, more than half involved a meniscal tear. [1] One of the earliest descriptions of the menisci was recorded by Bland-Sutton in 1897. At that time, the menisci were thought to be vestigial tissue and were depicted as "the functionless remnants of intraarticular leg muscles." [2] Further advances in our understanding of the menisci have demonstrated that the menisci provide mechanical support and secondary stabilization, localized pressure distribution and load sharing, and lubrication and propioception to the knee joint.[3] Arnoczky and Warren first described the blood supply for each meniscus. [1] They illustrated a microvascular perimeniscal plexus supplied by the vascularized synovial tissue on the periphery of the menisci. This plexus is formed from the medial and lateral geniculate arteries from the popliteal artery. The vascular supply terminates in short capillaries that extend from the periphery inwards, constituting approximately $10 \%$ to $30 \%$ of the width of the medial meniscus and $10 \%$ to $25 \%$ of the width of the lateral meniscus. Vascular supply to the meniscus determines its potential for repair. The experimental findings have demonstrated that the peripheral meniscal blood supply is capable of producing a reparative response similar to that observed in other connective tissues.[4]

Numerous classifications of tears of the menisci have been proposed based on location or type of tear, etiology, and other factors. Whereas it is recognized that tears are more common when degenerative changes, cystic formations, or congenital anomalies are present, most of the commonly used classifications are according to the type of tear found at surgery. These are (1) longitudinal tears, (2) transverse and oblique tears, (3) combination of longitudinal and transverse tears (complex tears), (4) tears associated with cystic menisci, and (5) tears associated with discoid menisci.[5] Traditionally, meniscal tears were managed with meniscectomy. However, since the long-term morbidities of meniscus removal became apparent (eg, early development of knee osteoarthritis), management has been increasingly focused on meniscal repair. Tears of greater than one centimeter in length oriented in a vertical longitudinal direction and located in the periphery of the meniscus fall into the repairable category.[6]

The most common criteria for meniscal repair include a vertical longitudinal tear greater than $1 \mathrm{~cm}$ in length located within the vascular zone. Tears in the red-red zone (1-3 mm from the menisco-synovial junction) and red-white zone (3-5 mm from the menisco-synovial junction) have excellent healing potential. The tear also should be unstable and displaceable into the joint. In addition, the patient should be active and less than 40 years old. The knee should be either stable or will be stabilized with a ligamentous reconstruction simultaneously. Finally, the bucket handle portion and the remaining meniscal rim should be in good condition.[5]

Arthroscopically-aided meniscal repair was first reported by Ikeuchi, Grant et al., [7,8] Choi et al, Wang et al, and others have reported high rates of success with meniscal repair. $[9,10]$

In this research we will show our experience in Sohag University Hospital, Egypt in meniscal repair, using all-inside technique. The aim of the thesis was to study the capability of meniscal healing using all-inside meniscal repair system implants available by different arthroscopic techniques.

\section{Patients and methods:-}

This study was conducted during the period between January 2015 and June 2019, the main objective was to evaluate the results of arthroscopic meniscal repair using all-inside meniscal repair systems. Cases were selected on an outpatient basis. A detailed history and clinical assessment were done with special emphasis on traumatic or occupational injuries. Forty cases with suspected meniscal tears had a MRI done. Thirty cases were selected for diagnostic arthroscopy, and meniscal repair was decided only in twenty cases, while the other ten cases were excluded due to associated ligamentous injuries, deformities, bad quality menisci or irreparable tears.

Mean age was 26.5 years (range 20-39). They were 16 males, and four females. The majority of meniscal injuries (75\%) were caused by sport injuries with other causes like laborer work or a fall with a twist during walking accounting for five cases. Time interval between injury and surgery was recorded. Four patients were operated upon two weeks post-injury, two patients four weeks, 8 patients in six weeks time post-injury and 6 patients in eight weeks time post-injury. 
Ten cases showed wasting of the thigh muscles, especially the quadriceps muscle. Fifteen cases complained from their right knee of which thirteen cases were right leg dominant. Sixteen out of the twenty cases had medial compartment pain). Clinical evaluation for patients were done according to international knee documentation committee (IKDC). [12] Pre-operative findings were summarized in table 1.

\begin{tabular}{|c|l|l|l|l|}
\hline I. ITEM & Pain & Swelling & Locking & Range of motion \\
\hline II. NORMAL & $\begin{array}{l}0 \text { cases } \\
(0 \%)\end{array}$ & 5 cases $(25 \%)$ & 4 cases $(20 \%)$ & $\begin{array}{l}0 \text { cases } \\
(0 \%)\end{array}$ \\
\hline 1$) \quad$ Mild & $\begin{array}{l}2 \text { cases } \\
(10 \%)\end{array}$ & 10 cases $(50 \%)$ & 10 cases $(50 \%)$ & 5 cases $(25 \%)$ \\
\hline Moderate & 15 cases $(75 \%)$ & 5 cases $(25 \%)$ & 4 cases $(20 \%)$ & $\begin{array}{l}11 \text { case } \\
(55 \%)\end{array}$ \\
\hline Severe & 3 cases $(15 \%)$ & $\begin{array}{l}0 \text { cases } \\
(0 \%)\end{array}$ & 2 cases $(10 \%)$ & $\begin{array}{l}4 \text { cases } \\
(20 \%)\end{array}$ \\
\hline
\end{tabular}

Table 1:-Pre-operative clinical findings

Clinical examination include a variety of special tests to asses knee structural integrity, e.g, McMurray test, joint line tenderness (JLT), squat test, valgus/varus stress test, Lachman test, anterior and posterior drawer tests, and pivot shift test.

Preoperative plain radiographs and MRI were done for every patient, Stroller MRI grading system for meniscal tears was used.

Although repairable cases had to fall under the grade (3) group, some cases that were interpreted on the MRI as being a grade (2) meniscal lesions were found to be repairable tears communicating with at least one articular margin during arthroscopy.

Operative techniques:

Arthroscopic setup and positioning:

All patients were given spinal regional anesthesia. Limb exanguinated and a cotton-padded upper-thigh pneumatic tourniquet was applied. Stressing the knee to open up the various compartments is necessary for diagnostic or operative procedures. Careful knee assessment under anesthesia was crucial to rule out instability and/or confirm the preoperative diagnosis. Skin of the affected knee was carefully shaved just before surgery. thereafter the leg was prepared and draped properly.

\section{Diagnostic arthroscopy:}

The basic portals were used in all 20 cases for diagnostic arthroscopy,menisci were probed carefully from both upper and lower surfaces and any tear, no matter how small or relatively insignificant, was taken into consideration and well-planned for. Reducible bucket handle longitudinal full thickness peripheral tears were identified, reduced (if displaced into the notch; and a repair technique was decided. For irreducible fibrosed displaced bucket-handle tears, usually due to long-term neglected cases, a partial meniscectomy was done and were excluded from the study. Other degenerative tears not amenable for repair or those in the inner avascular third of the meniscus were dealt with either by partial meniscectomy if they caused pain and disability, or else left alone if less than one centimeter and stable with no symptoms and excluded from the study. All repaired menisci had good meniscal quality. Degenerative, small $(<10 \mathrm{~mm})$ symptomatic but stable menisci were debrided and minimally trimmed and left alone to delay the onset of osteoarthritis and excluded from the study. Articular cartilage was carefully inspected for any degenerative changes that would definitely affect the postoperative prognosis of the patient. Two cases with cartilage softening were detected during probing, one of them was localized to the femoral condyle on the side of meniscal repair, while the $2^{\text {rd }}$ case affected both tibial and femoral cartilage of both knee compartments, simple illustration of diagnostic arthroscopy is shown in Fig. 1.

Methods used for meniscal repair:

Identified repairable meniscal tears were first tested for reducibility by manipulating the displaced meniscus from under the femoral condyle back to its place using a probe or a grasper. Re-displacement of the meniscus was noted indicating either an unstable tear or a fibrosed meniscus. The edges of the tear were then debrided with the shaver. A rasp was then introduced through the working portal to abrade and freshen the edges to promote healing. Although no 
frank bleeding was seen while rasping, probably due to the use of a tourniquet, yet it was routinely done for all 20 cases (Fig.1).

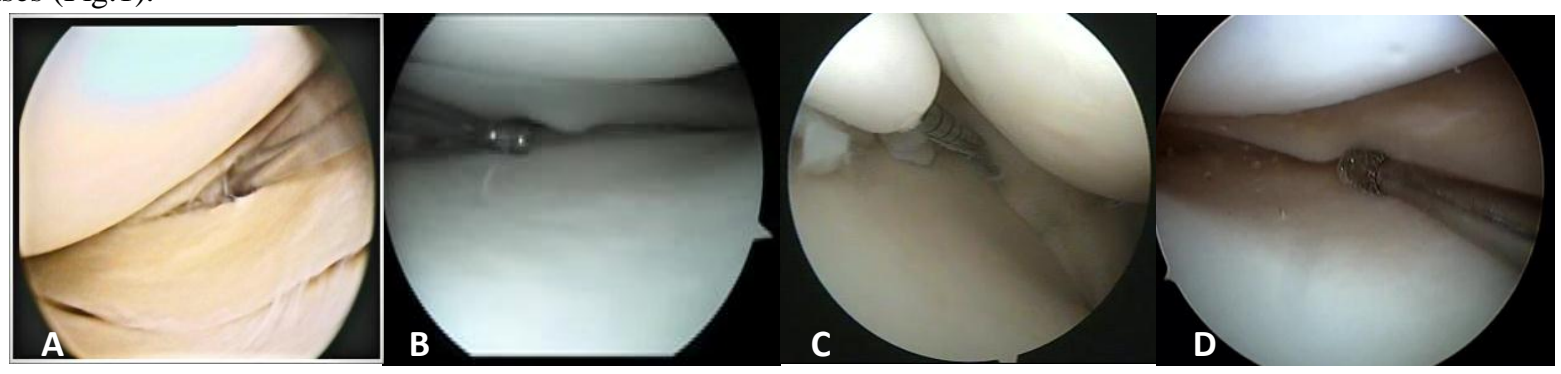

Figure 1: Diagnostic Arthroscopy, scope view

1. Displaced bucket handle meniscal tear

2. Probing

3. measuring the length of the tear by special gauge.

4. Rasping

Measuring the length of the meniscus from the estimated site of entry of the needle of the implant to the far periphery of the meniscus determined the required length ensuring good grip and purchase of the needle in the meniscal substance. A graduated measuring probe was introduced through the working portal and its tip placed at the meniscocapsular junction. The reading on the probe is then recorded at the estimated entry point of the implant putting in mind that a good purchase in the meniscal substance is a must (usually mid-way between the tear site and the inner border of the meniscus).

Deciding the number of implants to be used relied on the length of the tear. For example a 30-mm tear would take from 2 to 3 implants with 10 to $15 \mathrm{~mm}$ space in-between. After meniscal repair was done, the knee was irrigated with saline and the meniscus is re-probed to confirm adequate fixation and stability. The knee is then washed-out as many times as required, portals and any skin incision are then sutured with Vicryl 2-0, cleaned and covered by sterile dressing. A crepe bandage on top of cotton padding is wrapped gently around the knee before the tourniquet is released to minimize hemarthrosis. Figures $2 \& 3$ illustrate consequent steps of meniscal repair.

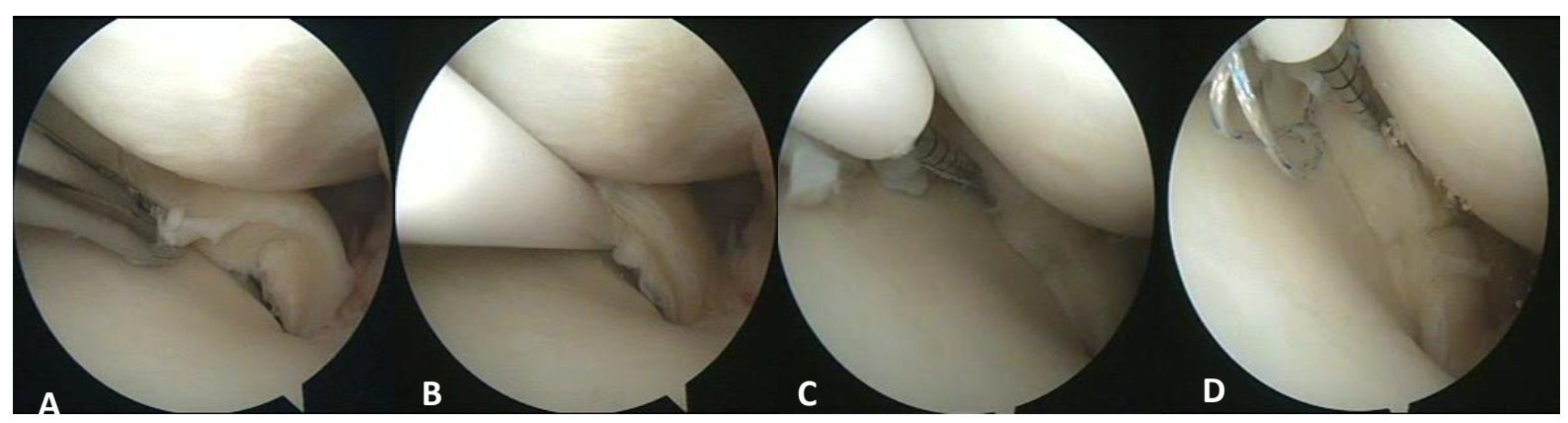

Figure 2:Preparation for meniscal repair, scope view

1. Preparation of the tear is important- Freshen the tear with a rasp or shaver

2. Pass the needle through the Meniscal substance and into the pericapsular tissue posteriorly

3. Insert the applier through a standard portal

4. Maintain pressure on the gun to prevent kickback. Use needle and silicone tubing to reduce the tear

\section{Postoperative care and rehabilitation}

Immediate postoperative:

No vacuum suction was used since all cases were isolated meniscal tears. All patients were discharged immediately postoperative and instructed to start an oral $1^{\text {st }}$ generation cephalosporin six hourly for ten days, +/- an analgesic antiinflammatory medication to alleviate postoperative pain if any. Before discharge, patients were prohibited from any weight bearing or range of motion for the first 2 weeks postoperative. During this period, patients immediately started isometric quadriceps exercises as well as straight leg raises.

Weight bearing and Knee protection: 
Rehabilitation was carried out cautiously and gradually for a minimum period of 6 months to avoid any stress on the repair site. Based on experimental studies, it was shown that meniscal healing with fibro-vascular scar tissue can take place by 10 weeks however, modulation of this scar into normal appearing fibro-cartilage could take up to several months.

Knee protection from weight bearing (by the use of crutches) was most important immediately postoperatively and for the first 6 weeks because at this early stage of healing, the initial scar is still developing and the only structure fixating the repair is the suture-tissue interface, which is a weak link.

No weight bearing whatsoever was allowed during the $1^{\text {st }} 2$ weeks. Touch ground weight bearing was gradually permitted thereafter with the aid of crutches till the end of the $6^{\text {th }}$ week after which gradual full weight bearing without crutches was begun.

Knee protection in a brace or in an elastic support immediately postoperative and only for the $1^{\text {st }} 2$ weeks was instructed after which it was removed to prevent unwanted quadriceps inhibition and allow early passive range of motion. Nine out of 20 cases used a hinged knee brace whereas the remaining 11 did not provide the brace and used a crepe bandage or an elastic knee support instead.

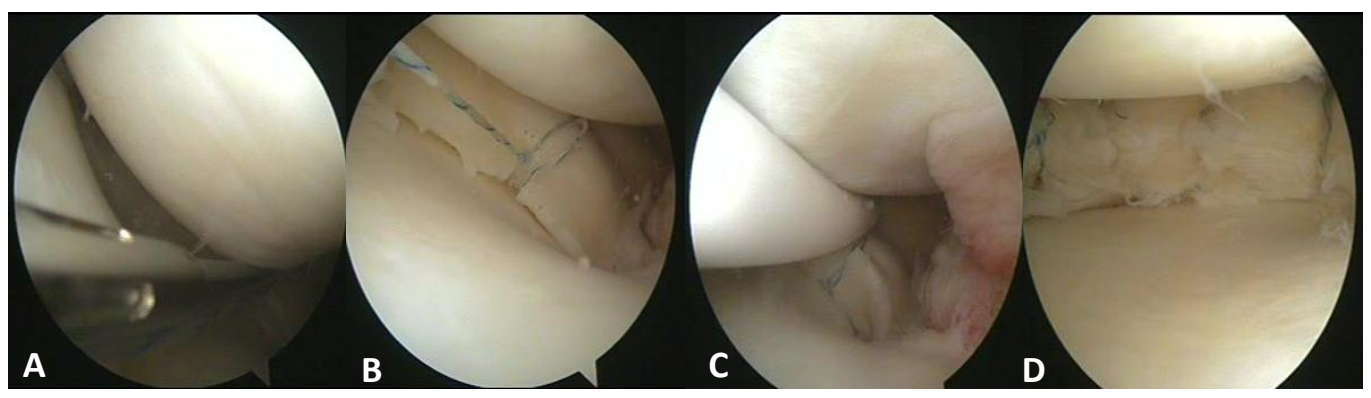

Figure 3:Serial steps of meniscal repair, scope view

1. Remove needle and applier from joint

2. Pull on the limb of suture to assure capture and fixation of the backstop

3. Using the arthroscopic pusher slide the knot. Pull on the suture with slightly more tension to avoid slack in the suture

4. Cut the limb of suture Repeat with additional implants Probe to assure tear is stable

\section{Range of motion}

Passive range of motion is helpful in reducing hemarthrosis, preventing joint capsule and hamstrings contracture, and maintaining nutrition to the healing meniscus. More important is that during passive motion, light forces are applied to the meniscus without the abrasive shear forces that are associated with active motion ${ }^{9}$.

Knees were kept fully extended for the $1^{\text {st }}$ two weeks with no range allowed after which very gradual passive range was started. At the beginning of the $7^{\text {th }}$ week, active range was started but at first without reaching full extension to avoid unwanted stresses on the repaired meniscus.

\section{Muscle strengthening}

Active quadriceps and hamstring strengthening exercises were not allowed before the beginning of the $3^{\text {rd }}$ month postoperatively to avoid undesirable stresses on the repair at an early stage. Post operative rehabilitation program is summarized in table 2.

\section{Postoperative evaluation:}

Clinical evaluation:

Full examination of the knee was done for every patient on the instructed dates for follow-up at the outpatient clinic. They included testing for pain, effusion, locking and range of motion, as well as some special tests that were tolerated like the Mac-Murray test and joint line tenderness.

\section{Postoperative plain radiographs:}

Routine standard postoperative radiographs were ordered for every patient every two months. It helped detect any decrease in the joint space as well as any other problems that may arise throughout the rehabilitation period.

\section{Postoperative MRI:}


MRI was ordered for patients at the 10th-15th month postoperatively to look either for meniscal healing with scar formation or failure. Failure was suspected when there was a gapping or displacement of the tear once again. MRI also helped figuring out any effusion or subchondral bone edema due to degenerative changes in the joint.

Postoperative evaluation arthroscope:

A second look arthroscopy was done for five patients after 15-20 months following the repair who had variable residual complaints like pain, locking, limited range or effusion. Findings were compared to their postoperative MRI findings.

\section{Results:-}

Mean operative time was $49.75+/-9$ min. Mean IKDC Score before was $40.25+8.35$, while the mean score postoperative was $84+13.92$.

Tear was in the body and posterior third of the meniscus in 15 case, in three cases was on was in the body only while in two cases was in the posterior horn. Zone of tear was reported as: 1) Mid-substance tears (middle 1/3) accounting for 4 cases, 2) peripheral tears (peripheral 1/3) accounting for 14 cases, and 3) menisco-capsular separation accounting for 2 cases. Tear length was recorded as: $15-30 \mathrm{~mm}$ in 3 cases, $10-15 \mathrm{~mm}$ in $13 \mathrm{cases}, 5-10 \mathrm{~mm}$ in 4 cases . Regarding the tear location (11 cases) were in the red red zone while (9 cases) were in the red white zone.

Postoperative plain radiographs:

Standard radiographs were ordered for all patients postoperatively to evaluate any articular or bony changes. Two cases showed narrowed joint space. Both cases need rescoping and show degenerative changes and failed repair.

\section{Postoperative MRI:}

Post operative MRI showed complete healing in seven case, partial healing in nine cases and failure to heal in four cases.

\section{Postoperative evaluation arthroscopy:}

A second look arthroscopy was done for five cases. These were the cases that had residual clinical problems or started to complain after a period of clinical improvement. Of all the clinical symptoms, locking with a limitation in range as well as severe pain were indications for a 2 nd look arthroscopy. A meniscus was considered healed if the residual cleft was $<10 \%$ of meniscal thickness, partially healed if the residual cleft was $<50 \%$ of the meniscal thickness and failed repair if the residual cleft $>50 \%$ of meniscal thickness. Articular cartilage as well as the condition and quality of the meniscus were assessed.

Four of five re-scoped cases revealed failed repairs (residual cleft $>50 \%$ of meniscal thickness). One of the five rescoped cases showed a partially healed tear (residual cleft $<50 \%$ of meniscal thickness) but was misinterpreted by MRI as a grade (3) tear. Failed repairs were treated with partial meniscectomy while partially healed one were continued on physiotherapy.

Speaking of meniscal quality after repair, two of the re-scoped knees (five knees) showed fibrotic changes. Stability, in the four failed repairs, was assessed and showed mild instability only when probed as well as no history of postoperative complaint of locking or limitation of the range.

\begin{tabular}{|c|c|c|c|c|c|c|c|}
\hline Item & $\begin{array}{c}\mathbf{1}^{\text {st }} \\
\text { weeks }\end{array}$ & $\begin{array}{c}\mathbf{2}^{\text {nd }} \\
\text { weeks }\end{array}$ & $\begin{array}{c}\mathbf{3}^{\text {rd }} \\
\text { weeks }\end{array}$ & $\begin{array}{c}\mathbf{4}^{\text {th }} \\
\text { weeks }\end{array}$ & $\begin{array}{c}\mathbf{3}^{\text {rd }} \\
\text { month }\end{array}$ & $\begin{array}{c}\mathbf{4}^{\text {th }} \\
\text { month }\end{array}$ & $\begin{array}{c}\mathbf{5}^{\text {th }} \\
\text { month + }\end{array}$ \\
\hline $\begin{array}{c}\text { Wearingt } \\
\text { beach } \\
\text { ground } \\
\text { extension } \\
\text { crutches }\end{array}$ & No & $\begin{array}{l}\text { Touch } \\
\text { ground } \\
\text { crutches }\end{array}$ & $\begin{array}{c}\text { Gradual } \\
\text { without } \\
\text { crutches }\end{array}$ & Yes & Yes & Yes \\
\hline $\begin{array}{c}\text { Knee } \\
\text { support }\end{array}$ & Yes & No & No & No & No & No & No \\
\hline ROM Ex. & $\begin{array}{c}0 \\
\text { degrees. }\end{array}$ & $\begin{array}{l}0-80 \\
\text { degree } \\
\text { max. } \\
\text { (passive). }\end{array}$ & $\begin{array}{l}0-100 \\
\text { degrees } \\
\text { max. } \\
\text { (passive). }\end{array}$ & $\begin{array}{l}20 \\
120 \text { degrees } \\
\text { (active) }\end{array}$ & $\begin{array}{c}\text { Full } \\
\text { range } \\
\text { (active) }\end{array}$ & $\begin{array}{c}\text { Full } \\
\text { range } \\
\text { (active) }\end{array}$ & $\begin{array}{l}\text { Full } \\
\text { range } \\
\text { (active) }\end{array}$ \\
\hline $\begin{array}{c}\text { Isometric } \\
\text { quadriceps ex. }\end{array}$ & Yes & Yes & Yes & Yes & Yes & Yes & Yes \\
\hline $\begin{array}{c}\text { Muscle } \\
\text { (quad. }\end{array}$ & No & No & No & No & Yes & Yes & Yes \\
\hline
\end{tabular}




\begin{tabular}{|c|c|c|c|c|c|c|c|}
\hline $\begin{array}{l}\text { hams.) } \\
\text { strengthening } \\
\text { with weights }\end{array}$ & & & & & & & \\
\hline $\begin{array}{l}\text { Stationary } \\
\text { ex. Bicycle }\end{array}$ & No & No & No & No & Yes & Yes & Yes \\
\hline $\begin{array}{cc}\text { Fast } \\
\text { walking } \\
\text { running }\end{array}$ & No & No & No & No & No & Yes & Yes \\
\hline $\begin{array}{c}\text { Sports } \\
\text { full activity }\end{array}$ & No & No & No & No & No & No & Yes \\
\hline
\end{tabular}

Table 2:-Detailed post operative rehabilitation program.

\section{Discussion:-}

Meniscal repair has become increasingly popular over the past two decades thanks to the better understanding of the biomechanical and functional properties of the intact meniscus in relation to knee mechanics and patient outcomes. However, the repair techniques require longer time to master, but postoperative results compared to meniscectomy are much more better, especially on long term follow up.[12]

Meniscus-to-femoral condyle congruity is essential for the development of circumferential hoop stresses and meniscus function. To restore the physiological relationship between femur, meniscus and tibia, accurate positioning of the meniscus based on precise meniscal sizing is critical in meniscus. [13]

Long term results after major meniscectomy were disappointing so that partial meniscectomy was advocated, a decrease in contact area of $10 \%$ increases the contact stresses by $65 \% .9$ Studies reporting the natural history of complete meniscectomy support preservation of as much meniscus as possible.[14]

Meniscal preservation is emphasized, as the menisci play important roles in weight bearing, stabilization and energy absorption. Recently, a shift towards meniscal preservation has lead to the development of new surgical techniques.[15]

The zone of tear in reference to blood supply is a major factor affecting the results of meniscal repair. Ramp lesions or red-red zone tears are expected to heal more readily than are those in the red-white zone. Many investigators have shown no difference in healing between red-red and red-white zone tears.[16]

The studied cases were in the middle age ranging from 20 to39 years (mean $=26.5+4.98)$. Statistically, age showed no significant difference as regards failure rate of repairs. In a similar study done by Barber and Herbert age was considered in the clinical evaluation of patients with symptomatic torn menisci. Seventy-two percent of their patients were 45 years or less. Therefore, the majority of the patients were of an age where repair was possible. Yet, no significant effect of age was shown on the healing rates of repaired menisci.[16] However, Becker et al. found that in older patients, most meniscal tears were of a degenerative nature and thus not amenable to repair. [4]

Concerning the sex of patients, $80 \%$ were males whereas only $20 \%$ were females. Two of the 5 symptomatizing (rescoped) cases were females, and both were failed repair. In a study conducted by Barrett et al. on 37 cases, $70.3 \%$ of the cases $(n=26)$ were males while only $29.7 \%(n=11)$ were females. The authors didn't mention any correlation between sex and the clinical outcome. The same results were stated by Eggli et al. $[18,19]$

The studied cases were selected with a body mass index (BMI) ranging from 20 to $31 \mathrm{~kg} / \mathrm{m}^{2}$ (mean $\left.=24.25+2.49\right)$ in an effort to exclude overweight patients that could jeopardize the success rate of the repairs. Statistically, BMI showed no significant difference as regards failure rate of repairs. Coggon et al found that overweight population is at high risk of developing osteoarthritis if a partial meniscectomy was to be performed. He also stressed at the reduction of body weight as a way to decreasing the burden on the knee. People undergoing meniscectomy or repair were the focus for his advice. [20]

As regards the time elapsed from injury, it was between 2 to 8 weeks in all cases with a mean of $5.6+2.21$ weeks. $80 \%$ of the 5 clinically symptomatizing cases came for repair 8 weeks post-injury, $(n=1)$ came after 2 weeks. These findings show that the duration elapsed since injury has its effect on the outcome of the repair yet statistically insignificant. Cameron and Saha found that the time from injury to repair did affect the healing rates of meniscal 
repair. [15] Barrett et al. in a study on 37 cases found that patients with older tears had a higher failure rate than those with acute tears. $22 \%$ of their cases repaired after 8 weeks from injury were clinical failures as compared with only $11 \%$ of repairs performed within 8 weeks from injury, yet statistically it was not significant. [18]

In this study, the assessment of the success rate was based mainly on clinical data including pain, locking, range of motion, effusion and muscle atrophy. The clinical tests used were the McMurray test and joint line tenderness. Imaging studies (X-ray and MRI) were also used. A second look arthroscopy was done for persistently symptomatic cases.

The overall clinical success rate in this study was $75 \%$ (15 repairs), which after re-scoping of the 5 symptomatic cases, success rate increased to $80 \%(\mathrm{n}=16)$. Henning et al. stated that clinical follow-up has the advantage of being readily available, but it raises the issue of including asymptomatic failures as successful repairs. [21]

According to the clinical data in this study, muscle atrophy was observed in the affected knees as compared with the normal side for a period of six months postoperatively. Preoperatively, 50\% $(n=10)$ had muscle atrophy. On postoperative follow-up, 7 cases showed improvement while only three cases persisted to have wasting in addition to 2 new cases with persistent symptoms that did not have atrophy preoperatively. The $25 \%(\mathrm{n}=5)$ with postrehabilitation atrophy were the cases who needed a second look arthroscopy due to persistent symptoms. Although clinical difference was obvious pre- and postoperatively, statistically there was no significant difference.

As regards pain, $75 \%$ of cases $(n=15)$ had no residual pain. On severe exertion, $20 \%(n=4)$ had mild pain while on minimum exertion, $5 \%(n=1)$ had moderate pain and no case had severe pain. Three of the 4 cases with mild pain and this with moderate pain were re-scoped revealing failed repairs.

No swelling was detected at follow-up in $65 \%$ of the cases $(n=13)$. On severe exertion, $20 \%$ ( $n=4)$ had minimal effusion and $10 \%(\mathrm{n}=2)$ had moderate effusion while $5 \%$ had severe effusion on minimal exertion. Rest and icing relieved the effusion in 2 cases with mild and one with moderate effusion. The remaining cases with persistent swelling were re-scoped. According to McDowell et al. the benefits of cold therapy in the management of the orthopedic patients include pain control with less narcotic usage and decreased edema that allows for earlier rehabilitation. [22]

In this study, $100 \%$ of cases $(n=20)$ had not encountered any locking following the repair.

Range of motion was fully regained in $75 \%$ of operated knees $(n=15)$. Limited range was observed in $25 \%$ of cases, being mild in $20 \%(\mathrm{n}=4)$ and moderate in $5 \%(\mathrm{n}=1)$ of the cases. Re-scoping for 3 of the 4 cases with mild limitation showed healing in one case and failure in the other 2 cases. Their limited range was probably due to the associated effusion. The case with moderate limitation showed failed unstable repair.

The grades for each symptomatology were further re-grouped into normal (no complaint) and abnormal group (the rest of the grades). Accordingly, 25\% of cases had persistent pain on follow-up as compared to $100 \%$ painful knees preoperatively. $35 \%$ had residual swelling postoperatively as compared to $75 \%$ of cases with knee effusion preoperatively. $25 \%$ of cases had limited range of motion postoperatively as compared to $100 \%$ with different degrees of range limitations preoperatively. Although the aforementioned data shows clear clinical difference between the pre- and postoperative results, yet statistically, it showed non-significant difference for all.

Plain radiographs were used in this study to determine the width of the joint space. Narrowed joint space on postoperative radiographs was found to be positive in two cases $10 \%$. One of two cases shows articular cartilage degeneration at time of repair, the 2 cases were among the rescoped cases with no SD. However, Barrett et al. stated that articular cartilage degeneration evident at the time of the meniscal repair did not have any significant effect on the outcome of the meniscal repair. [18]

In this study tear zone showed $20 \%$ with mid-zone tears, $70 \%$ with peripheral tears and $10 \%$ with menisco-capsular tears. Four failed repairs of all cases were peripheral tears and only one was a menisco-capsular separation of no SD. Considering the fact that only the peripheral $10 \%$ to $30 \%$ of the adult meniscus retains its vascularity. Barrett et al. showed that the location of the tear is one of the most important factors influencing the healing rates of meniscal repairs.[18] 
In this study, 4 of 5 re-scoped cases revealed failed repairs (residual cleft $>50 \%$ of meniscal thickness. One of the five re-scoped cases showed a partially healed tear (residual cleft $<50 \%$ of meniscal thickness). Failed repairs were treated with partial meniscectomy while partially healed ones were continued on physiotherapy and considered healed repairs.

As regards the failure rate, in this study the failure rate is $20 \%$ for the all cases. on the other hand in a study done by Nepple et al. the failure rate of inside-out meniscal repair ranged from $0 \%$ to $26.9 \%$. Pooled analysis of inside-out meniscal repair revealed a failure rate of $22.3 \%$. While the failure rates of all-inside meniscal repair were $4.0 \%$, $28.4 \%$, and $28.6 \%$. Pooled analysis of all-inside meniscal repair revealed a failure rate of $24.3 \%$ Nepple et al., showing no statistical difference.[23] degeneration evident at the time of the meniscal repair did not have a significant effect on the outcome of the repair.

\section{Conclusion:-}

The essential goal of meniscal repair is protecting the knee from long term degenerative changes by the cushion like and stabilizing effect of the menisci.

Having found no difference in surgical outcome between different types of all-inside meniscal repair techniques, we investigated other factors such as age \& sex, injury location, length, zone \& site of tear for their effect.

Patient age and time elapsed from injury was recorded for every case being important factors that might affect repair outcome dramatically. Patient's weight (body mass index) was also recorded as a factor that could jeopardize the repair.

MRI, despite its fallacies, was a major diagnostic tool that helped tear identification as well as exclusion of ligamentous injuries and other associated knee problems.

\section{References:-}

1. Catherene Laible, Drew A. Stein, Daniel N. Kiridly, Meniscal repairAAOSApril 2013, Vol 21, No 4

2. Bland Sutton (1879), The semilunar cartilages. Br J Surg; 29(116):407- 114.

3. Maak TG,Fabricant PD andWickiewicz TL. Indicationsformeniscusrepair. Clin Sports Med.2012; 31(1):1-14.

4. Becker R, Starke C, Heymann M and Nebelung W. Biomechanical properties under cyclic loading of seven meniscus repair techniques. Clin. Orthop, 2002; 400: 236-245.

5. Miller MD. Revision cruciate ligament surgery with retention of femoral interference screws. Arthroscopy, $1998 ; 14$ (1): 111-114.

6. De-Haven KE and Bronstein RD. Arthroscopic medial meniscal repair in the athlete. Clin. Sports Med., 1997; 16: 6986.

7. Ikeuchi H. (1979): Meniscus surgery using the Watanabe arthroscope. Am. Orthop. Clin. North; 10: 629.

8. Grant JA,Wilde J,Miller BS andBedi A.Comparisonofinside-outandall-insidetechniquesfor therepairofisolatedmeniscaltears: a systematic review. Am J Sports Med.2012 Feb

9. Choi NH,Kim TH andVictoroff BN. Comparison of arthroscopic medial meniscal suture repair techniques: inside-out versus all-inside repair.Am J Sports Med.2009; 37(11):2144-50 .

10. Wang KH,Hwang DH,Cho JH,Changale SD,Woo SJ andNha KW. Arthroscopic direct repair for a complete radial tear of theposteriorrootof themedialmeniscus. ClinOrthop Surg.2011; 3(4):332-5.

11. Ahmed KM, Said HG, Ramadan EKA, El-Radi MA \& El-Assal MA (2019) Arabic translation and validation of three knee scores, Lysholm Knee Score (LKS), Oxford Knee Score (OKS), and International Knee Documentation Committee Subjective Knee Form (IKDC). SICOT-J, 5, 6.

12. Vascellari A, Rebuzzi E, Schiavetti S and Colleti N. all inside meniscal repair using fast fix meniscal repair systeme : is still needed to avoid weight bearing? Asystematic review. Musculoskletal Surg.2012:96(3):149-54

13. Beamer BS,Masoudi A,Walley KC,Harlow ER,Manoukian OS,Hertz B,Haeussler C,Olson JJ,Deangelis JP,Nazarian AandRamappa AJ. Analysisof anewall-insideversusinside-outtechniqueforrepairingradialmeniscaltears. Arthroscopy.2015; 31(2):293-8.

14. Vanderhave KL,Moravek JE,Sekiya JK andWojtys EM.Meniscus tears in the young athlete: results of arthroscopic repairJPediatrOrthop.2011; 31(5):496-500.

15. Cameron JC and Saha S. Meniscal allograft transplantation for unicompartmental arthritis of the knee. ClinOrthop, 1997; 164-171.

16. Choi NH,Kim TH andVictoroff BN. Comparison of arthroscopic medial meniscal suture repair techniques: inside-out versus all-inside repair.Am J Sports Med.2009; 37(11):2144-50 . 
17. Barber FA and Herbert MA (2000): Meniscal Repair Devices. Arthroscopy Assocciation of North America.

18. Barrett GR, Field MH, Treacy SH and Ruff CG. Clinical results of meniscus repair in patients 40 years and older. Arthroscopy, 1998; 14(8): 824-829.

19. Eggli S, Wegmuller H, Kosina J, Huckell C and Jakob RP (1995): Long-term results of arthroscopic meniscal repair: An analysis of isolated tears. Am. J. Sports Med., 23 (6): 715-720.

20. Coggon, D.; Reading, I.; Croft, P.; McLaren, M.; Barrett, D. and Cooper, C. (2001): Knee osteoarthritis and obesity. Int. J. Obes. Relat. Metab. Disord.,25(5): 622-627.

21. Henning, C.E.; Lynch, M.A. and Clark, J.R. (1987): Vascularity for healing of meniscus repairs. Arthroscopy, 3: 1318.

22. McDowell, J.H.; McFarland, K.G. and Nalli, B.J. (1994): Use of Cryotherapy for Orthopeadic Patients. Orthopedic Nursing, 13 (5): 21-30.

23. Nepple JJ,Dunn WR andWright RW. Meniscalrepairoutcomesatgreaterthanfiveyears: asystematicliteraturereviewandmeta-analysis. J Bone Joint Surg Am.2012; 94(24):2222-7. 